\title{
Flare Occurrence in the Solar Active Regions with Reversed Helicity Sign
}

\author{
S. D. Bao, G. X. Ai, and H. Q. Zhang
}

Beijing Astronomical Observatory/National Astronomical Observatories, Chinese Academy of Sciences, Beijing 100012, China

\begin{abstract}
Based on the Huairou Solar Observing Station dataset, we computed the current helicity for several hundreds of active regions and found that: (1) Active regions that do not follow the hemispheric helicity sign rule show more flare activity than normal active regions. (2) The relative number of active regions with reversed helicity sign is higher near sunspot maximum. (3) It appears that during solar cycle 22 the southern hemisphere has more the reversed-sign active regions and stronger flare activity than the northern hemisphere.
\end{abstract}

\section{Introduction}

Observations have revealed that a hemispheric preference of net sign of helicity, or handedness, exists throughout the solar atmosphere - in the photosphere, the chromosphere, the corona, and the solar wind (e.g., Bao, Ai, \& Zhang 2000; and references therein). In the photosphere more than $70 \%$ of active regions in the northern/southern hemisphere have negative/positive current helicity $\left(H_{\mathrm{c}}\right.$ or $\alpha$ ). Zhang and Bao (1999) have found the active regions with reversed helicity sign tend to emerge at certain longitudes. Current helicity of magnetic fields also plays an important role in solar flares (Pevtsov et al. 1995, 1996; Bao et al. 1999). Canfield et al. (1999) have classified active regions according to morphology (sigmoidal or non-sigmoidal) and nature of activity (eruptive or non-eruptive), and have found that regions are significantly more likely to be eruptive if they are either sigmoidal or large. The aim of this paper is to statistically examine whether active regions with abnormal helicity sign show more flare activity.

\section{Data Analysis and Results}

The data we used for this study were obtained with the vector video magnetograph at the Huairou Solar Observing Station of Beijing Astronomical Observatory. The detailed description of this instrumentation and the observational technique may be found elsewhere (Wang et al. 1996; Bao et al. 2000). All vector magnetograms were observed under good weather and seeing conditions during the time when the active regions were located near the central meridian.

Bao and Zhang (1998) used the same dataset to calculate current helicity of 422 active regions in the cycle 22 . They found that $84 \%$ of the active regions 


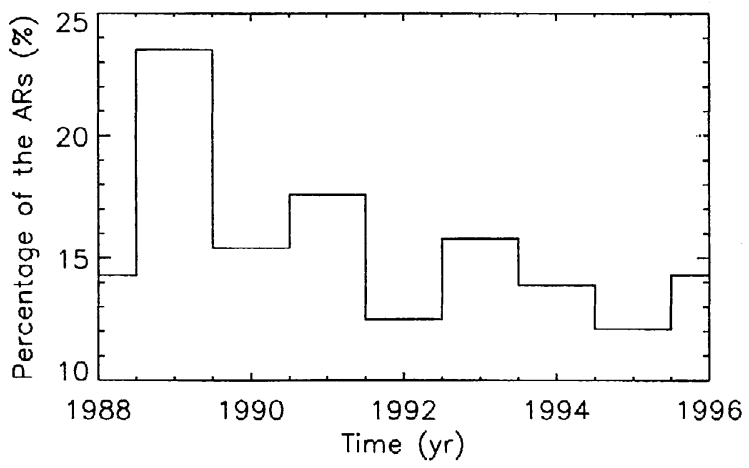

Figure 1. Distribution of the percentage of active regions that do not follow the helicity sign rule appeared on the solar disk with time.

in the northern hemisphere have negative helicity, and $79 \%$ in the southern hemisphere have positive helicity. The active regions that disobey this rule are listed in Table 1 including information on their flare activity. Most of the regions accompany by abundant flare activity. As a comparison, we also analyzed all active regions that follow the helicity sign rule, and found that active regions almost without flare activity amount to $25 \%$ of all normal active regions, but only $12 \%$ for the active regions with reversed helicity sign. In other words, these regions with reversed helicity sign have a tendency for enhanced flare activity.

Table 1 also shows that the number of active regions with reversed helicity sign reaches maximum near sunspot maximum (1989 and 1991), like other indicators of activity (such as flare count). Since the productivity of active regions is also high around sunspot maximum, we display a histogram of active regions with reversed helicity sign as a fraction of total number of active regions that appeared on the solar disk during the cycle 22 (Figure 1). This indicates that during the maximum years of solar activity the physical processes in deeper layers of the Sun are extremely complicated and dynamic, which leads to the emergence of a large number of tubes of different twisting, along with the appearance of relatively larger number of active regions with reversed helicity sign.

In addition, the north-south asymmetry can also be found in Table 1 from both the number of the reversed-sign active regions and the frequency of powerful flares occurred in these regions. Such a hemispheric asymmetry may be useful for understanding the physical conditions and processes deep below the photosphere. One should study further the relationship between flares and active regions with abnormal helicity using much larger dataset.

Acknowledgments. We are indebted to the staff of the Huairou Solar Observing Station for the observations. We also thank A.A. Pevtsov for his reading of this manuscript. This work is supported by National Science Foundation of China under grants 19791090 and 19973008. 
Table 1. Synopsis of flare occurrence in the active regions with reversed helicity sign. ' $\mathrm{l}$ ' - having some large flares exploded in an active region, 's' only smaller flares occurred, ' $m$ ' - appearing many flares, ' $f$ ' - a few flares, and '-' - almost no flares.

\begin{tabular}{|c|c|c|c|c|c|}
\hline NOAA/AR & Date & Coordinate & $\bar{H}_{c}$ & Flare class & Productivity \\
\hline \multicolumn{6}{|c|}{ Northern hemisphere } \\
\hline 5451 & 19 Apr., 1989 & N10 W05 & + & $\mathrm{s}$ & $\mathrm{m}$ \\
\hline 5569 & 29 Jun., 1989 & N21 W04 & + & $\mathrm{s}$ & m \\
\hline 5589 & 17 Jul., 1989 & N27 E04 & + & $\mathrm{s}$ & f \\
\hline 5676 & 10 Sep., 1989 & N27 W02 & + & $\mathbf{s}$ & f \\
\hline 5769 & 04 Nov., 1989 & N25 E05 & + & $\mathrm{s}$ & $\mathrm{m}$ \\
\hline 5776 & 08 Nov., 1989 & N17 W01 & + & 1 & $\mathrm{~m}$ \\
\hline 5796 & 23 Nov., 1989 & N11 W05 & + & - & - \\
\hline 5821 & 08 Dec., 1989 & N20 E04 & + & $\mathrm{s}$ & m \\
\hline 6095 & 10 Jun., 1990 & N23 E00 & + & $\mathrm{s}$ & $\mathbf{m}$ \\
\hline 6150 & 12 Jul., 1990 & N07 W06 & + & $\mathbf{s}$ & $\mathbf{m}$ \\
\hline 6199 & 15 Aug., 1990 & N13 W04 & + & $\mathrm{s}$ & $\mathrm{m}$ \\
\hline 6605 & 02 May , 1991 & N09 E01 & + & $\mathrm{s}$ & f \\
\hline 6792 & 23 Aug., 1991 & N03 E01 & + & - & - \\
\hline 6893 & 30 Oct., 1991 & N17 E02 & + & $\mathrm{s}$ & f \\
\hline 6908 & 10 Nov., 1991 & N21 E05 & + & $\mathrm{s}$ & f \\
\hline 7019 & 21 Jan., 1992 & N17 W02 & + & $\mathrm{s}$ & $\mathrm{m}$ \\
\hline 7029 & 29 Jan., 1992 & N17 E01 & + & - & - \\
\hline 7050 & 12 Feb., 1992 & N11 W02 & + & $\mathrm{s}$ & $\mathbf{m}$ \\
\hline 7145 & 27 Apr., 1992 & N12 W04 & + & $\mathrm{s}$ & f \\
\hline 7425 & 18 Feb., 1993 & N15 W03 & + & 1 & $\mathrm{~m}$ \\
\hline 7448 & 17 Mar., 1993 & N16 W04 & + & 1 & $\mathrm{~m}$ \\
\hline 7496 & 10 May, 1993 & N15 W06 & + & $\mathrm{s}$ & $\mathrm{m}$ \\
\hline 7512 & 30 May , 1993 & N08 W05 & + & $\mathbf{s}$ & f \\
\hline 7671 & 20 Feb., 1994 & N11 W04 & + & $\mathrm{s}$ & $\mathrm{m}$ \\
\hline 7794 & 31 Oct., 1994 & N13 E05 & + & $\mathrm{s}$ & $\mathbf{m}$ \\
\hline 7870 & 14 May , 1995 & N10 E02 & + & $\mathrm{s}$ & $\mathrm{m}$ \\
\hline \multicolumn{6}{|c|}{ Southern hemisphere } \\
\hline 5137 & 19 Aug., 1988 & S20 E00 & - & $\mathbf{s}$ & f \\
\hline 5148 & 17 Sep., 1988 & S11 E03 & - & $\mathrm{s}$ & f \\
\hline 5212 & 04 Nov., 1988 & S17 W06 & - & $\mathrm{s}$ & $\mathbf{m}$ \\
\hline 5312 & 13 Jan., 1989 & S32 E04 & - & 1 & $\mathrm{~m}$ \\
\hline 5497 & 23 May, 1989 & S20 E01 & - & 1 & $\mathrm{~m}$ \\
\hline 5622 & 05 Aug., 1989 & S27 E01 & - & 1 & $\mathrm{~m}$ \\
\hline 5657 & 23 Aug., 1989 & S20 E08 & - & - & - \\
\hline 5669 & 05 Sep., 1989 & S17 E01 & - & 1 & $\mathrm{~m}$ \\
\hline 5747 & 20 Oct., 1989 & S26 W01 & - & 1 & $\mathrm{~m}$ \\
\hline 5801 & 26 Nov., 1989 & S20 E00 & - & - & - \\
\hline 5947 & 26 Feb., 1990 & S17 W04 & - & $\mathrm{s}$ & $\mathrm{m}$ \\
\hline 6050 & 09 May , 1990 & S11 E03 & - & $\mathrm{s}$ & f \\
\hline 6064 & 19 May , 1990 & S15 W01 & - & 1 & $\mathbf{m}$ \\
\hline 6131 & 03 Jul., 1990 & S21 W02 & - & 1 & $\mathrm{~m}$ \\
\hline 6227 & 26 Aug., 1990 & S23 W02 & - & 1 & $\mathrm{~m}$ \\
\hline 6462 & 27 Jan., 1991 & S18 E06 & - & $\mathrm{s}$ & $\mathrm{m}$ \\
\hline 6509 & 24 Feb., 1991 & S21 E03 & - & $\mathrm{s}$ & f \\
\hline 6593 & 21 Apr., 1991 & S12 W06 & - & 1 & $\mathrm{~m}$ \\
\hline 6615 & 06 May , 1991 & S11 W04 & - & $\mathrm{s}$ & $\mathrm{m}$ \\
\hline 6698 & 30 Jun., 1991 & S05 W04 & - & - & - \\
\hline 6818 & 04 sep., 1991 & S12 W02 & - & 1 & $\mathrm{~m}$ \\
\hline 6873 & 15 Oct., 1991 & S23 W07 & - & $\mathrm{s}$ & $\mathrm{m}$ \\
\hline 7026 & 27 Jan., 1992 & S13 E00 & - & $\mathrm{s}$ & f \\
\hline 7039 & 04 Feb., 1992 & S15 W03 & - & 1 & $\mathrm{~m}$ \\
\hline
\end{tabular}


Table 1. - Continued

\begin{tabular}{cccccc}
\hline NOAA/AR & Date & Coordinate & $H_{c}$ & Flare class & Productivity \\
\hline 7335 & 12 Nov., 1992 & S18 W06 & - & $\mathrm{s}$ & $\mathrm{f}$ \\
7345 & 23 Nov., 1992 & S24 W04 & - & $\mathrm{l}$ & $\mathrm{m}$ \\
7400 & 13 Jan., 1993 & S09 W04 & - & $\mathrm{s}$ & $\mathrm{m}$ \\
7411 & 26 Jan., 1993 & S12 E01 & - & $\mathrm{s}$ & $\mathrm{m}$ \\
7493 & 04 May, 1993 & S10 W03 & - & $\mathrm{s}$ & $\mathrm{f}$ \\
7563 & 17 Aug., 1993 & S04 W04 & - & $\mathrm{s}$ & $\mathrm{m}$ \\
7625 & 30 Nov., 1993 & S15 W01 & - & - & - \\
7629 & 08 Dec., 1993 & S21 E00 & - & $\mathrm{s}$ & $\mathrm{m}$ \\
7742 & 03 Jul., 1994 & S09 E00 & - & $\mathrm{s}$ & $\mathrm{f}$ \\
7792 & 24 Oct., 1994 & S08 E04 & - & $\mathrm{s}$ & $\mathrm{f}$ \\
7815 & 14 Dec., 1994 & S11 W05 & - & $\mathrm{l}$ & $\mathrm{m}$ \\
7842 & 20 Feb., 1995 & S14 E06 & - & - & - \\
7854 & 21 Mar., 1995 & S17 W02 & - & $\mathrm{s}$ & $\mathrm{m}$ \\
7921 & 12 Nov., 1995 & S10 E01 & - & $\mathrm{s}$ & $\mathrm{f}$ \\
7999 & 26 Nov., 1996 & S05 E05 & - & $\mathrm{s}$ & $\mathrm{m}$ \\
\hline
\end{tabular}

\section{References}

Bao, S. D., Ai, G. X., \& Zhang, H. Q. 2000, J. Astrophys. Astr., in press

Bao, S. D., Pevtsov, A. A., Wang, T. J., \& Zhang, H. Q. 2000, Solar Phys., 195, 75

Bao, S. D. \& Zhang, H. Q. 1998, ApJ, 496, L43

Bao, S. D., Zhang, H. Q., Ai, G. X., \& Zhang, M. 1999, A\&AS, 139, 311

Canfield, R. C., Hudson, H. S., \& McKenzie, D. E. 1999, Geophysical Research Letters, 26, 627

Pevtsov, A. A., Canfield, R. C., \& Metcalf, T. R. 1995, ApJ, 440, L109

Pevtsov, A. A., Canfield, R. C., \& Zirin, H. 1996, ApJ, 473, 533

Zhang, H. Q. \& Bao, S. D. 1999, ApJ, 519, 876

Wang J. X., Shi Z. X., Wang H. N., \& Lü Y. P. 1996, ApJ, 456, 861 